\title{
Formation and applications of periodic structures in transparent materials induced by single fs laser beam
}

\author{
Jianrong Qiu \\ State Key Laboratory of Luminescent Materials and Devices, South China University of Technology, China \\ E-mail address: qjr@scut.edu.cn
}

\begin{abstract}
In this paper, we present our recent research development on single fs laser beam induced periodic structures, i.e. nanovoid array, tilted grating structures, nanograting etc, and discuss the mechanisms and promising applications of the observed periodic structures.
\end{abstract}

Periodic structures can be used as grating and lens etc. Usually they are prepared by point-by-point or line by line laser scanning and using multi-beam laser interference technique. However, periodic nanovoid array was observed in transparent materials along the propagation direction of single fs laser beam. ${ }^{[1]}$ By adopting a physical model which combines the nonlinear propagation of fs laser pulses with the spherical aberration (SA) effect at the interface of two mediums of different refractive indices, reasonable agreements between the simulation results and the experimental results were obtained. By comparing the fluence distributions of the case with both SA and nonlinear effects included and the case with only consideration of SA, we suggest that SA, which results from the refractive index mismatch between air and fused silica glass, is the main reason for the formation of the self-organized void array. ${ }^{[2]}$ We observed self-organized microgratings in the bulk $\mathrm{SrTiO}_{3}$ crystal by scanning the laser focus in the direction perpendicular to the laser propagation axis. The groove orientations of those gratings could be controlled by changing the irradiation pulse number per unit scanning length, which could be implemented either through adjusting the scanning velocity at a fixed pulse repetition rate or through varying the pulse repetition rate at a fixed scanning velocity. ${ }^{[3]}$ The possible formation mechanism of the self-organized microgratings was also discussed.

In 1999, Kazansky et al observed fs laser induced polarization-dependent light scattering in Ge-doped silica glass. ${ }^{[4]}$ Qiu et al. observed so-called memorized polarization-dependent emission in various glasses and crystals, and predicted the permanent polarization-dependent micro-structure induced by fs laser. ${ }^{[5]}$ Shimotsuma et al. first demonstrated the nanograting structures, and since then there have been extensive investigations on the formation of nanograting structures. ${ }^{[6-7]}$ We demonstrated the inscription of polarization dependent self- organized nanogratings inside fused silica by $1 \mathrm{KHz}$ fs laser. The results were confirmed by optical birefringence and scanning electron microscope. We showed that self-organized nanogratings can be fabricated within a wide range of pulse energy and a certain scanning speed range with $1 \mathrm{KHz}$ fs laser. Direct writing of polarizer was demonstrated by formation of a plane consisting of many lines through scanning the fs laser induced nanograting structure. Interestingly, rotated 3D self-organized nanograting was observed inside fused silica by using a $250 \mathrm{KHz}$ fs laser. ${ }^{[8]} \mathrm{The}$ difference of birefringence signal of non-reciprocal writing lines indicates that the excited pulses may possess a finite tilt of intensity front, which produces an electric field vector parallel to the incident direction to modulate the excited electron plasma. We suggest that the orientation of nanograting depends on the correlation between the polarization plane azimuth and the tilted intensity front based on previous investigations by Kazansky et al.

\section{References}

[1] S. Kanehira, J. Si, J. Qiu, K. Fujita, K. Hirao, “, "Periodic nanovoid structures via femtosecond laser irradiation”, Nano Lett., 5, 1591-95 (2005).

[2] J. Song, X. S. Wang, X. Hu, Y. Dai, J. Xu, J. Qiu, Y. Cheng, Z. Xu, "Formation mechanism of self-organized voids in dielectrics induced by tightly focused femtosecond laser pulses", Appl. Phys. Lett., 92, 92904-906 (2008).

[3] J. Song, X. Wang, X. Hu, Y. Cheng, Z. Xu, J. Qiu, "Orientation-controllable self-organized microgratings induced in the bulk SrTiO3 crystal by a single femtosecond laser beam", Opt. Express, 15, 14524-26 (2007).

[4] P. Kazansky, H. Inouye, T. Mitsuyu, K. Miura, J. Qiu and K. Hirao, “Anomalous anisotropic light scattering in Ge-doped silica glass", Phys. Rev. Lett., 82, 2199-2202 (1999).

[5] J. Qiu, P. Kazansky, J. Si, K. Miura, T. Mitsuyu, K. Hirao and A. L. Gaeta, "Memorized polarization dependent light scattering in rare-earthion-doped glass", Appl. Phys. Lett., 77, 1940-42 (2000). 
[6] Y. Shimotsuma, P. Kazansky, J. Qiu, and K. Hirao, "Self-organized nanogratings in glass irradiated by Ultrashort light pulses”, Phys. Rev. Lett., 91, 247405-07 (2003).

[7] C. Hnatovsky, E. Simova, P. Rajeev, D. Rayner, P. Corkum, R. Taylor, "Femtosecond laser writing of porous capillaries inside fused silica glass", Opt. Lett., 32, 1459-61 (2007).

[8] Y. Dai, G. Wu, X. Lin, G. Ma, J. Qiu, "Femtosecond laser induced rotated 3D self-organized nanograting in fused silica”, Opt. Express, 20, 18072-75 (2012). 